\title{
In vivo pharmacological study on the effectiveness of available polyclonal antivenom against Hemiscorpius lepturus venom
}

\author{
Jalali A (1, 2), Pipelzadeh M H (3, 2), Seyedian R (4), Rahmani A H (5), Omidian N (6
}

(1) Department of Pharmacology and Toxicology, School of Pharmacy, Jundishapur University of Medical Sciences, Ahvaz, Iran; (2) Toxicology Research Center, Jundishapur University of Medical Sciences, Ahvaz, Iran; (3) Department of Pharmacology, School of Medicine, Jundishapur University of Medical Sciences, Ahvaz, Iran; (4) Department of Pharmacology and Toxicology, University of Medical Sciences, Bushehr, Iran; (5) Department of Internal Medicine, Razi Hospital, Jundishapur University of Medical Sciences, Ahvaz, Iran; (6) Faculty of Veterinary Medicine, Chamran University, Ahvaz, Iran.

\begin{abstract}
The available Razi Institute antivenom is still, empirically, used by intramuscular (IM) administration for the treatment of scorpion stings in humans by six medically dangerous species including Hemiscorpius lepturus ( $H$. lepturus). The aim of this study was to assess the neutralizing ability and effectiveness of the antivenom in inhibiting hemoglobinuria, biochemical changes, increased microalbuminuria and urinary lactate dehydrogenase (LDH) following $H$. lepturus sting. Simultaneous intramuscular administration of $10 \mu \mathrm{L}$ and $100 \mu \mathrm{L}$ of antivenom, after 24 hours, had no significant preventive effect on the extent and degree of hemoglobinuria or proteinuria produced in venom-treated rats. After IM administration of antivenom, no significant changes in decreased red blood cell (RBC) count and hemoglobin were observed. Immediate intramuscular administration of $10 \mu \mathrm{L}$ of antivenom had no significant effects on both LDH and microalbuminuria. The present findings did not present correlation with clinical signs. Therefore, to fully assess the efficacy of the available antivenom and make appropriate recommendations, more in vivo or in vitro investigations including antigen-antibody interaction, enzymatic analysis and route-dependent administration are required.
\end{abstract}

Key words: Hemiscorpius lepturus, scorpion, venom, hemolysis, antivenom.

\section{INTRODUCTION}

Scorpion envenomation is characterized by various symptoms such as pain, sweating, fever and hypertension. In more severe cases, these symptoms are followed by pulmonary edema and myocardial damage that can be fatal (1). In Khuzestan province, Iran, envenomation by Hemiscorpius lepturus is a major concern during warm months of the year. Although only $12 \%$ of all the scorpion sting cases in Khuzestan province are due to this species, it is responsible for more than $95 \%$ of deaths. The mortality rate due to $H$. lepturus stings has occasionally reached more than $8 \%$ in recent years, particularly among pediatric patients (2).
Following H. lepturus sting, various degrees of local toxicity are observed in most of the cases including macular erythema, purpuric changes, bullae, necrosis, and ulcers (3). The neurological effects of $H$. lepturus venom are similar to those of Buthidae scorpions. However, red blood cell (RBC) lysis induced by $H$. lepturus venom is more significant than other scorpion toxins (4). Moreover, different investigations showed hemotoxicity including hemoglobinuria, microscopic hematuria, proteinuria or microalbuminuria and/or their fragments on renal function. H. lepturus sting provokes acute renal failure at a frequency great enough to suggest a causal relationship between renal failure and RBC lysis in humans (4-7). 
Hemolysis due to envenomation by this scorpion is so common that when a patient is suspected of being stung by a scorpion, the first clinical test is to check the presence of hemoglobin in the patient's urine. Severe hemolysis and secondary renal failure due to scorpion stings are frequently observed in Khuzestan province, southwestern Iran, complications that, to our knowledge, have not been properly observed in other scorpion stings elsewhere.

In Iran, serotherapy with multivalent antivenom against six common Iranian scorpions is the most frequently used therapy in the treatment of scorpion stings over the last 30 years $(8,9)$. Antivenom is capable of reverting hematological effects caused by the Indian red scorpion (Mesobuthus tamulus) (10). Despite the increased annual number of envenomation cases in this area and the results of clinical and experimental tests, there is no consensus among clinicians on the effectiveness of this type of treatment following envenomation by $H$. lepturus. Furthermore, no previous toxicopharmacological study has specifically focused on the effectiveness of antivenom in neutralizing the toxic manifestations of $H$. lepturus venom.

Therefore, the aim of present study was to assess the effectiveness of antivenom therapy in preventing the toxic effects of $H$. lepturus venom in envenomed rats using different blood and kidney toxicity markers in vivo.

\section{MATERIALS AND METHODS}

\section{Animals}

Male adult Wistar rats (200-300 g) were purchased from the animal house of Jundishapur Medical University, Ahvaz, Iran. Animals were housed individually in aluminum cages $(60 \mathrm{x}$ $45 \times 15 \mathrm{~cm}$ ) and allowed to feed on a standard, commercial, pellet diet (Shushtar Khorakdam Co., Iran) supplemented with fresh vegetables and tap water ad libitum. Animal house temperature was set at $23 \pm 2^{\circ} \mathrm{C}$ and a 12 -hour light/dark cycle was maintained during the study.

Animal procedures were in accordance with the guidelines for animal care prepared by Committee on Care and Use of Laboratory Animal Resources, National Research Council, USA.

\section{H. lepturus}

Lyophilized $H$. lepturus venom was supplied by Razi Institute of Iran (Hessarak, Karaj). Briefly, $H$. lepturus scorpions were trapped and their venoms were collected by electrical stimulation on their telsons $(15 \mathrm{~V})$. The collected venom was pooled, lyophilized and stored at $-20^{\circ} \mathrm{C}$ before use. For the experiment, an aliquot of the lyophilized venom was reconstituted by addition of distilled water or phosphate buffer saline solution (PBS). Protein content of the venom and antivenom was determined by Coomassie blue dye-binding method with bovine serum albumin as standard (11).

\section{Iranian Polyvalent Antivenom Preparation}

The multivalent scorpion antivenom (5$\mathrm{mL}$ ampoules, stored at 2 to $8^{\circ} \mathrm{C}$ ) is a pepsindigested refined and concentrated preparation obtained from equine hyperimmune serum and, according to the manufacturer information, it has neutralizing potency against the venoms of the six endemic Iranian scorpions (Androctonus crassicauda, Buthotus saulcyi, Buthotus schach, Odontobuthus doriae, Mesobuthus eupeus and $H$. lepturus). The polyvalent scorpion antivenon neutralizes the venom (not less than ten mice $\mathrm{LD}_{50}$ ) of the aforementioned six Iranian scorpions (8).

\section{In Vivo Time Profile of Hemoglobinuria and Proteinuria Induced by $H$. lepturus and Assessment of Preventive Effects of Antivenom in Rats}

Ten micrograms of $H$. lepturus venom diluted in $100 \mu \mathrm{L}$ of PBS was injected subcutaneously into three of four groups of rats $(n=5)$. Two doses ( 10 and $100 \mu \mathrm{L}$ ) of antivenom were intramuscularly injected immediately in the opposite leg of the first two groups. The third group (positive group) was injected with $100 \mu \mathrm{L}$ of normal saline. The fourth group (placebo treated negative control) was injected with normal saline followed by 100 $\mu \mathrm{L}$ of antivenom. During the next 24 hours, the animals were kept in a metabolic cage and had free access to water and food pellets. At various time intervals (namely 1, 3, 6, 12, 18 and 24 hours) the collected urine was tested for the presence of hemoglobinuria and proteinuria by urinary dipstick (Medi-Test Combi $\mathrm{II}^{\oplus}$, Medisave, UK). The minimum sensitivity of the test strip for detection of hemoglobinuria and proteinuria was respectively $0.015 \mathrm{mg} / \mathrm{dL}$ and $10 \mathrm{mg} / \mathrm{dL}$. 
In Vivo Assessment of Antivenom on Biochemical Changes Induced by $\boldsymbol{H}$. lepturus Venom

In a separate series of experiments, the same experimental protocol on animal selection and grouping as in vivo time profile of hemoglobinuria and proteinuria part were carried out. Twenty four hours later, blood samples were obtained by cardiac puncture, under anesthesia (pentobarbital sodium, $50 \mathrm{mg} /$ $\mathrm{kg}$ ). The collected blood samples were transferred to the laboratory for $\mathrm{RBC}$ count (RBC in $\mathrm{x} 10^{6}$ / $\mu \mathrm{L})$, hemoglobin $(\mathrm{Hb}, \mathrm{g} / \mathrm{dL})$, serum potassium $\left(\mathrm{K}^{+}, \mathrm{mEq} / \mathrm{L}\right)$, blood urea nitrogen (BUN, mg/ $\mathrm{dL})$, serum creatinine $(\mathrm{Cr}, \mathrm{mg} / \mathrm{dL})$, prothrombin time (PT, seconds) and partial thromboplastin time (PTT, seconds).

\section{In Vivo Preventive Effects of Antivenom on Nephrotoxic Properties of $\boldsymbol{H}$. lepturus in Rats}

To assess the potential for tubulopathy and proteinuria of the venom and the protective effects of antivenom, LDH enzyme and diurnal microalbuminuria levels were measured $(12,13)$. For this purpose, three groups of rats $(n=5)$ were selected and $10 \mu \mathrm{g}$ of $H$. lepturus venom diluted to $100 \mu \mathrm{L}$ (with PBS) was injected subcutaneously in two groups via insulin syringe. Immediately after venom administration, $10 \mu \mathrm{L}$ of polyvalent antivenom, diluted to $100 \mu \mathrm{L}$ (with PBS) was injected intramuscularly in the treated group. Normal saline was injected subcutaneously in the control group which also received antivenom. Each group was maintained in different cages with free access to food and water. During the following 24 hours, urine was collected for measurement of microalbumin and LDH enzyme using ELISA method with Pars Azmoon kits (Iran).

\section{Statistical Analysis}

The results are expressed as mean \pm SD. Paired Student's t-test and analysis of variance followed by Tukey's test were used to determine the significant differences between means; $\mathrm{p}<$ 0.05 was considered statistically significant.

\section{RESULTS}

\section{In Vivo Assessment of Hemoglobinuria and Proteinuria Induced by $\mathrm{H}$. lepturus in Rats}

A total of three rats developed hemoglobinuria 24 hours after venom administration [one after 12 hours $(1+)$, and the other two after 18 hours (2+)]. Furthermore, after 18 hours three rats showed proteinuria $(1+)$. Simultaneous intramuscular administration of 10 and $100 \mu \mathrm{L}$ of antivenom, after 24 hours, had no significant preventive effect on both the extent and degree of hemoglobinuria or proteinuria.

Effect of Antivenom on Biochemical Changes

Table 1. Effectiveness of two doses $(10 \mu \mathrm{L}$ and $100 \mu \mathrm{L})$ of immediately administered IM antivenom on biochemical and hemolytic changes induced by SC injection of $H$. lepturus venom ( $n=5$ in each group)

\begin{tabular}{c|c|c|c|c}
\hline & Control & $V(10 \mu \mathrm{g})$ & $\begin{array}{c}\mathrm{V}(10 \mu \mathrm{g})+\mathrm{AV} \\
(10 \mu \mathrm{L})\end{array}$ & $\begin{array}{c}\mathrm{V}(10 \mu \mathrm{g})+\mathrm{AV} \\
(100 \mu \mathrm{L})\end{array}$ \\
\hline PT (s) & 12.8 & 13.2 & 13.11 & 13.31 \\
\hline PTT (s) & 21.8 & 22.2 & 21.17 & 22.56 \\
\hline BUN (mg/dL) & 10.44 & 11.52 & 10.56 & 11.24 \\
\hline $\mathrm{Cr}(\mathrm{mg} / \mathrm{dL})$ & 1.01 & 1.13 & 1.17 & 1.21 \\
\hline Serum K+ (mEq/L) & 4.42 & 4.58 & 4.6 & 4.52 \\
\hline $\mathrm{Hg}(\mathrm{g} / \mathrm{dL})$ & 15.2 & $14.21^{*}$ & $14.24^{*}$ & $14.38^{*}$ \\
\hline $\mathrm{RBC}\left(\times 10^{6}\right)$ & 8.8 & $7.02^{*}$ & $7.11^{*}$ & $7.22^{*}$ \\
\hline
\end{tabular}

IM: intramuscular, AV: antivenom, V: venom, SC: subcutaneous.

${ }^{*} p<0.05$ between control and venom and venom + antivenom treated rats. ANOVA followed by Tukey's test $(n=5$ in each group) 
Table 2. Effectiveness of immediately administered IM antivenom on microalbuminuria and increase of urinary LDH enzyme following SC injection of $H$. lepturus venom-treated rats $(n=5)$; normal saline was administered in the control group instead of venom

\begin{tabular}{c|c|c|c}
\hline Treated group & Microalbuminuria (mg/24 h) & LDH (IU/L) & Urine volume (mL/24 h) \\
\hline $\mathrm{NS}$ & $18.4 \pm 2.18$ & $183 \pm 23.6$ & $16.2 \pm 3.4$ \\
\hline $\mathrm{V}(\mathbf{1 0} \boldsymbol{\mu g})$ & $180.8^{\mathrm{b}} \pm 7.47$ & $1173^{\mathrm{c}} \pm 137.1$ & $22.2 \pm 4.2$ \\
\hline $\mathrm{V}(\mathbf{1 0} \boldsymbol{\mu g})+\mathrm{AV}(\mathbf{1 0} \mu \mathrm{L})$ & $162.2^{\mathrm{b}} \pm 17.36$ & $919.4^{c} \pm 48.4$ & $20 \pm 2.5$ \\
\hline
\end{tabular}

IM: intramuscular, AV: antivenom, V: venom, SC: subcutaneous, NS: normal saline.

b $p<0.01$ and $c p<0.001$ between control and both venom and venom + antivenom treated rat groups. ANOVA followed by Tukey's test ( $n=5$ in each group).

\section{Induced by H. lepturus Venom in Rats}

Following administration of $10 \mu \mathrm{g}$ of $H$. lepturus venom, significant reductions in $\mathrm{RBC}$ count $\left(8.8\right.$ versus $\left.7.02 \times 10^{6} / \mu \mathrm{L}, \mathrm{p}<0.05\right)$ and hemoglobin $(15.2$ versus $14.21 \mathrm{~g} / \mathrm{dL}, \mathrm{p}<$ $0.05)$ were observed. However, there were no statistically significant differences on other factors (PT, PTT, BUN, Cr and serum $\mathrm{K}^{+}$) in envenomed and non treated groups. Following intramuscular administration of 10 and $100 \mu \mathrm{L}$ of antivenom, no significant changes in $\mathrm{RBC}$ count $\left(7.11\right.$ versus $\left.7.22 \times 10^{6} / \mu \mathrm{L}\right)$ and hemoglobin (14.24 versus $14.38 \mathrm{~g} / \mathrm{dL}$ ) were observed in antivenom-treated rats (Table 1).

In Vivo Assessment of Effectiveness of Antivenom against Changes in LDH and Microalbuminuria Induced by $\boldsymbol{H}$. lepturus Venom in Rats

Twenty four hours after envenomation with $10 \mu \mathrm{g}$ of $H$. lepturus venom, significant increases in microalbuminuria $(18.4 \pm 2.18$ versus $58.5 \pm$ $7.4 \mathrm{mg} / 24$ hours, $\mathrm{p}<0.01)$ and urinary lactate dehydrogenase $(183 \pm 23.6$ versus $1173.4 \pm$ 137.1 IU/L, p < 0.001) were recorded. Immediate intramuscular injection of $10 \mu \mathrm{L}$ of antivenom had no significant effects on increased LDH (tubulopathy) and microalbuminuria in rats (Table 2).

\section{DISCUSSION}

$H$. lepturus envenomation is a real health problem in Khuzestan. The multiorgan injury of victims admitted to intensive care units (ICU) is common. Despite the use of sophisticated diagnostic tools and aggressive management, blood and renal involvement remains the main problem in victims.

This study addresses the neutralizing ability of available polyspecific antivenom used for the treatment of recurrent clinical manifestations following stings of the most dangerous scorpion of Iran, H. lepturus (2).

In the present study, $10 \mu \mathrm{g}$ of $H$. lepturus venom and 10 and $100 \mu \mathrm{L}$ of antivenom were administered respectively via subcutaneous and intramuscular routes. Venom dose was adjusted according to the amount of scorpion venom injected. Moreover, 10 to $100 \mu \mathrm{L}$ of antivenom was administered to rats, since in the current Iranian protocol, two vials of $5 \mathrm{~mL}$ antivenom are recommended for an adult person (with $70 \mathrm{~kg}$ ). Intramuscular administration is commonly used in Iran. Although some clinicians believe that intravenous injection may cause anaphylactic shock, so they avoid this type of route, manufacturers believe that there is no side effect provoked by intravenous infusion. As a whole, there is no general consensus regarding dose, route of administration and its effectiveness in treatment of human envenomation by this scorpion. Moreover, concerning dose for both venom and antivenom, the design of this study was based on clinical practice and not on doseresponse experiments. So, the results may provide conclusive evidence from a clinical point of view. Therefore we did not consider all requirements for experimental designing such as in vitro incubation of venom with antivenom.

Our results showed that subcutaneous injection of this venom in rats caused mild hemoglobinuria and proteinuria after 18 hours. There was no azotemia or evidence of acute renal failure in rats by measurement of blood urea nitrogen and 
serum creatinine. Furthermore, estimation of PT and PTT revealed that coagulation parameters were within normal limits similar to those reported following envenomation by Loxosceles recluse in humans (14).

In a previous study, dog erythrocytes were found to be more susceptible than other animals to jellyfish venom (15). Similarly, in vitro hemolysis induced by $H$. lepturus venom was greater in cow than in other animals (horses, goats and chickens) (16). Our results showed that hemolysis induced by $H$. lepturus venom solution in rats was less potent than that reported for the venom of Loxosceles adelaida (17). These variations may reflect differences in the phospholipid composition of the erythrocyte plasma membrane of these species, as well as the accessibility of cellular membrane targets such as negative phospholipids to PLA 2 action (18-20).

Other explanations of these phenomena may include differences in binding and insertion of toxin molecules into plasma membrane followed by oligomerization to form transmembrane pores resulting in colloid osmotic lysis (21). The other possible mechanism that mediates RBC destruction is complement-mediated hemolysis or those mediated by sphingomyelinase II (20$21)$. The exact way by which $H$. lepturus induces hemolysis of $\mathrm{RBC}$ is not yet clearly defined and this deserves further pharmacodynamic and biochemical investigations.

Systemic disturbances, such as renal failure, hemolysis and other clinical manifestations, in envenomed patients by this scorpion may be attributable to enzymatic components (22). Interestingly, these enzymatic entities are relatively similar by their molecular weights to those of Loxosceles desserta (23). Hence, it is suspected that the enzymatic components of $H$. lepturus venom may play an indirect role in the activation of complement system, which participates in dermonecrosis and hemolysis of the envenomed patients as observed in loxocelism (24). The findings of Seyedian et al. (22) showed that the available antivenom has high affinity with $H$. lepturus venom, suggesting that the antivenon has specificity for detection and inhibition of the enzymatic activities of this venom (22). So we can propose and provide other conclusive evidence that different and appropriate routes of administration are for the neutralizing ability of antivenom.
There were no significant differences between envenomed rats and control in PT, PTT, BUN, $\mathrm{Cr}$ and serum $\mathrm{K}^{+}$. The underlying reason for ineffectiveness of antivenom in preventing toxic manifestations of the venom is not clear. However, it is possible that there are differences in the pharmacokinetics between venom and antivenom, as well as nonspecific binding of antivenom to serum proteins. Moreover, the administration of the recommended dose of antivenom intramuscularly is not able to properly ensure the neutralizing ability of antivenom. Prudently, this hypothesis needs to be assessed in further studies.

There is no general agreement among clinicians regarding the effectiveness of antivenom in treatment of envenomed patients (25-26). It is proposed that effective antivenom therapy must be applied as soon as possible intravenously, since this route is preferred regarding its higher neutralizing capacity, especially in severe cases of envenomation (27). However, this route also carries a greater risk of side effects, particularly possible unwanted anaphylactic shock. This dictates the production of pure and higher potent antivenom preparations that may overcome this limitation and improves treatment outcome (2829). It should be noted that intravenous infusion of scorpion antivenom is commonly employed elsewhere for envenomed patients (30).

Additionally, there are discrepancies among researchers concerning the effectiveness of administration of scorpion antivenom by intramuscular route for treatment of envenomed patients. For example, in a study performed on rats envenomed by Androctonus australis hector scorpion, cardiovascular and pulmonary effects were neutralized by this route of administration (31). However, in another experiment, IM administration of antivenom in envenomed patients by Buthus tunetanus occitanus did not show significant therapeutic effects (32).

Subcutaneous envenomation by $H$. lepturus venom produced hyperglycemia and augmentation in serum LDH in rabbits (4). Furthermore, microalbuminuria was considered an initial sign of renal damage, which predicts proteinuria in hyperglycemic status $(33,34)$. So, we used ELISA technique for measuring LDH and microalbuminuria, two markers that could not be measured by dipstick method, and were also proven to be reliable and robust for the detection of early renal damage to assess the 
severity of envenomation in rats (35). Our results expressed that subcutaneous injection induced significant increase in urinary LDH enzyme and microalbuminuria after 24 hours. Simultaneous intramuscular injection of antivenom did not prevent the production of tubulopathy and microalbuminuria in treated rats.

In the present study, significant serum hemoglobin, microalbuminuria and increase of urinary LDH enzymes were noticed after $H$. lepturus venom administration. These changes were not similar to those observed in experimental animals that received venom of the Buthidae family (36-37). Song et al. (37) suggested that Buthus martensii Karsch venom presents antithrombotic action through resistance against platelet aggregation. The present study demonstrated that $H$. lepturus has no effects on platelet aggregation. It is well known that venom counteracts platelet aggregation, and may affect $\mathrm{PT}$ and PTT time.

Since the antivenom was administered immediately after envenomation, these findings further confirm the suspicion that this antivenom is not a suitable preparation and further attempts need to be taken to prepare more specific antivenom for intravenous administration against this dangerous scorpion.

\section{CONCLUSION}

Considering the large number of envenomations by $H$. lepturus in rural and urban areas of southern provinces of Iran and their clinical consequences especially in children, our findings suggest that intramuscular route of antivenom administration is not a suitable method for treatment of envenomation by this scorpion. According to our methods, it seems that this venom is not fully neutralized through intramuscular route of administration. However, the lack of neutralizing activity observed in vivo can be considered a problem in antivenon neutralizing ability, and not in the route utilized. So, serotheraphy must be improved particularly regarding proteinuria and hemoglobinuria, both related to renal failure in patients. Prudently, to fully assess the effectiveness of the available antivenom, additional studies including in vitro evaluation, pharmacokinetics and routedependent administration of both venom and antivenom are required.

\section{ACKNOWLEDGEMENTS}

The authors would like to acknowledge the financial support by the Deputy of Research Affairs of Ahvaz Jundishapur University of Medical Sciences.

\section{COPYRIGHT}

(c) CEVAP 2011

\section{SUBMISSION STATUS}

Received: September 13, 2010.

Accepted: March 1, 2011.

Abstract published online: March 3, 2011.

Full paper published online: May 31, 2011.

\section{CONFLICTS OF INTEREST}

There is no conflict.

\section{FINANCIAL SOURCE}

The Deputy of Research Affairs of Ahvaz Jundishapur University of Medical Sciences provided the financial grants.

\section{ETHICS COMMITTEE APPROVAL}

Animal procedures were in accordance with the guidelines for animal care prepared by the Committee on Care and Use of Laboratory Animal Resources, National Research Council, USA. Moreover, the study was approved by the Ethics Committee of Jundishapur University of Medical Sciences.

\section{CORRESPONDENCE TO}

AMIR JALALI, Golestan Road, School of Pharmacy, Jundishapur University of Medical Sciences, Ahvaz, Iran. Phone: +98 6113738380 . Fax: +986113738381. Email: amjalali@hotmail. com.

\section{REFERENCES}

1. Nicholson GM, Graudins A, Wilson HI, Little MJ, Broad KW. Arachnid toxinology in Australia: clinical toxicology to potential applications. Toxicon. 2006; 48(7):872-98.

2. Jalali A, Pipelzadeh MH, Sayedian R, Rowan EG. A review of epidemiological, clinical and in vitro physiological studies of envenomation by the scorpion Hemiscorpius lepturus (Hemiscorpiidae) in Iran. Toxicon. 2010;55(2-3):173-9.

3. Radmanesh M. Cutaneous manifestations of the Hemiscorpius lepturus sting: a clinical study. Int J Dermatol. 1998;37(7):500-7.

4. Mirakabbadi A, Zolfagharian Z, Hedayat $\mathrm{H}$, 
Jalali A. Clinical and biochemical manifestation produced by scorpion (Hemiscorpius lepturus) venom in experimental animals. J Venom Anim Toxins incl Trop Dis. 2007;13(4):758-65.

5. Radmanesh M. Clinical study of Hemiscorpion lepturus in Iran. J Trop Med Hyg. 1990;93(5):32732.

6. Pipelzadeh MH, Dezfulian AR, Jalali MT, Mansouri AK. In vitro and in vivo studies on some toxic effects of the venom from Hemiscorpius lepturus scorpion. Toxicon. 2006;48(1):93-103.

7. Pipelzadeh $\mathrm{MH}$, Jalali A, Taraz M, Pourabbas R, Zaremirakabadi A. An epidemiological and clinical study on scorpionism by the Iranian scorpion Hemiscorpius lepturus. Toxicon. 2007;50(7):984-92.

8. Latifi M, Tabatabai M. Immunological studies on Iranian scorpion-venom and antiserum. Toxicon. 1979;17(6):617-20.

9. Tabatabayi M, Hedaiat A, Akbari A. A study of the geographical distribution of the scorpions in the south of Iran. Pajouhesh va Sazandegi. 1998;34(1):112-5.

10. Murthy KRK, Zare MA. The use of antivenom reverses hematological and osmotic fragility changes of erythrocytes caused by Indian red scorpion Mesobuthus tamulus concanesis Pocock in experimental envenoming. J Venom Anim Toxins. 2001;7(1):113-38.

11. Bradford MM. A rapid and sensitive method for the quantitation of microgram quantities of protein utilizing the principle of protein-dye binding. Anal Biochem. 1976;72(1):248-54.

12. Obatomi DK, Plummer DT. Renal damage caused by gentamicin: a study of the effect in vitro using isolated rat proximal tubular fragments. Toxicol Lett. 1995;75(1-3):75-83.

13. Anderson S, Diamond JR, Karnovsky MJ, Brenner BM. Mechanisms underlying transition from acute glomerular injury to late glomerular sclerosis in a rat model of nephrotic syndrome. J Clin Invest. 1988;82(1):1757-68.

14. Donepudi SK, Ahmed KA, Stocks RM, Nelson D, Thompson JW. Aural involvement in loxoscelism: case report and literature review. Int J Pediatr Otorhinolaryngol. 2005;69(11):1559-61.

15. Kang C, Munawir A, Cha M, Sohn ET, Lee H, Kim JS, et al. Cytotoxicity and hemolytic activity of jellyfish Nemopilema nomurai (Scyphozoa: Rhizostomeae) venom. Comp Biochem Physiol C Toxicol Pharmacol. 2009;150(1):85-90.

16. Salimian J, Zargan J, Ebrahimi F, Farahmandzad A, Hajibeygi A. Role of Hemiscorpius lepturus venom on red blood cell fragility. Kowsar Med J. 2002;7(3):185-90.

17. Pretel F, Goncalves-de-Andrade RM, Magnoli
FC, da Silva MER, Ferreira Jr JMC, van den Berg $\mathrm{CW}$, et al. Analysis of the toxic potential of venom from Loxosceles adelaida, a Brazilian brown spider from karstic areas. Toxicon. 2005;45(4):449-58.

18. Diaz C, Leon G, Rucavado A, Rojas N, Schroit AJ, Gutiérrez JM. Modulation of the susceptibility of human erythrocytes to snake venom myotoxic phospholipases $\mathrm{A}_{2}$ : role of negatively charged phospholipids as potential membrane binding sites. Arch Biochem Biophysics. 2001;391(1):5664.

19. Villegas E, Corzo G. Pore-forming peptides from spiders. Toxin Reviews. 2005;24(3):345-57.

20. Tambourgi DV, Pedrosa MF, de Andrade RM, Billington SJ, Griffiths M, van der Berg CW. Sphingomyelinases $\mathrm{D}$ induce direct association of $\mathrm{Clq}$ to the erythrocyte membrane causing complement mediated autologous haemolysis. Mol Immunol. 2007;44(4):576-82.

21. Bhakdi S, Tranum-Jensen J. Damage to cell membranes by pore-forming bacterial cytolysins. Prog Allergy. 1988; 40(1):1-43.

22. Seyedian R, Pipelzadeh MH, Jalali A, Kim E, Lee H, Kang C, et al. Enzymatic analysis of Hemiscorpius lepturus scorpion venom using zymography and venom-specific antivenin. Toxicon. 2010;56(4):521-5.

23. Barbaro KC, Knysak I, Martins R, Hogan C, Winkel K. Enzymatic characterization, antigenic cross-reactivity and neutralization of dermonecrotic activity of five Loxosceles spider venoms of medical importance in the Americas. Toxicon. 2005;45(4):489-99.

24. Espino-Solis GP, Riaño-Umbarila L, Becerril B, Possani LD. Antidotes against venomous animals: state of the art and prospectives. J Proteomics. 2009;72(2):183-99.

25. Abroug F, Elatrous S, Nouira S, Haguiga H, Touzi N, Bouchoucha S. Serotherapy in scorpion envenomation: a randomised controlled trial. Lancet. 1999;354(9182): 906-9.

26. Ismail $\mathrm{M}$. Treatment of the scorpion envenoming syndrome: 12-years experience with serotherapy. Int J Antimic Agents. 2003;21(2):170-4.

27. Theakston RDG, Warrell DA, Griffiths E. Report of a WHO workshop on the standardization and control of antivenoms. Toxicon. 2003;41(5):54157.

28. Krifi MN, Amri F, Kharrat $H$, El Ayeb M. Evaluation of antivenom therapy in children severely envenomed by Androctonus australis garzonii (Aag) and Buthus occitanus tunetanus (Bot) scorpions. Toxicon. 1999;37(11):1627-34.

29. Amitai Y. Clinical manifestations and management of scorpion envenomation. Public Health Rev. 1998;26(3):257-63. 
30. Ismail $M$. The treatment of the scorpion envenoming syndrome: the Saudi experience with serotherapy. Toxicon. 1994;32(9):1019-26.

31. Hammoudi-Triki D, Lefort J, Rougeot C, Robbe-Vincent A, Bon C, Laraba-Djebari F, et al. Toxicokinetic and toxicodynamic analyses of Androctonus australis hector venom in rats: optimization of antivenom therapy. Toxicol Appl Pharmacol. 2007;218(3):205-14.

32. Krifi MN, El Ayeb M, Dellagi K. The improvement and standardization of antivenom production in developing countries: comparing antivenom quality, therapeutical efficiency, and cost. J Venom Anim Toxins. 1999;5(2):128-41.

33. Bigazzi R, Bianchi S, Baldari D, Sgherri G, Baldari G, Campese VM. Microalbuminuria in salt-sensitive patients. A marker for renal and cardiovascular risk factors. Hypertension. 1994;23(2):195-9.
34. Mogensen CE. Microalbuminuria predicts clinical proteinuria and early mortality in maturity-onset diabetes. N Engl J Med. 1984;310(1):356-60.

35. El-Shitany NA, El-Haggar S, El-Desoky K. Silymarin prevents adriamycin-induced cardiotoxicity and nephrotoxicity in rats. Food Chem Toxicol. 2008;46(7): 2422-8.

36. Zare MA, Murthy KRK, Haghnazari L. Scorpion venom poisoning in experimental animals. Arch Inst Razi. 1994;44/45(1):67-72.

37. Song YM, Tang XX, Chen XG, Gao BB, Gao E, Bai L, et al. Effects of scorpion venom bioactive polypolypeptides on platelet aggregation and thrombosis and plasma 6-keto-PG F1alpha and TXB2 in rabbits and rats. Toxicon. 2005;46(2):2305. 